\title{
Experiments of the Pressure Drop of Propane Considering Hysteresis and Metastability
}

\author{
Xenia Gabrisch ${ }^{1}$, Jens-Uwe Repke ${ }^{1}$ \\ ${ }^{1}$ Chair of Process Dynamics und Control \\ Technical University of Berlin \\ Straße des 17. Juni 135, 10623 Berlin, Germany \\ xenia.gabrisch@tu-berlin.de; jens-uwe.repke@tu-berlin.de
}

\begin{abstract}
There is a broad database on two-phase pressure drop of refrigerants through capillaries in literature, since capillaries are a commonly used throttle device in heat pumps. However, the following essential aspects are insufficiently included: extensive data on natural refrigerants, precise capillary specifications, and the consideration of the hysteresis effect on vaporization delay and the metastable region of the flow, respectively. This paper contributes to a more comprehensive database of the two-phase pressure drop of propane with respect to the hysteresis curves that occur in the capillary. The present study investigates a copper capillary tube with $1 \mathrm{~m}$ length and $1.1 \mathrm{~mm}$ inner diameter. The manufacturer's specification of the capillary is refined to enable a more predictive numerical analysis of the pressure drop. Experimental data comprises a mass flux of $4300 \mathrm{~kg} / \mathrm{m}^{2} \mathrm{~s}$, a constant inlet pressure of 15 bar and subcooling temperatures ranging from $5{ }^{\circ} \mathrm{C}$ to $9{ }^{\circ} \mathrm{C}$. The presence and magnitude of the hysteresis becomes evident in the measurements: starting from a higher inlet temperature and increasing the subcooling temperature results in a higher pressure drop ( $\sim 5)$ than starting from a lower inlet temperature and decreasing the subcooling temperature. Additionally, experimental results show that a metastable region must be present that leads to a delay in vaporization and thereby affects the measured pressure drop.
\end{abstract}

Keywords: Two-Phase Pressure Drop, Propane, Metastable Region, Hysteresis Effect on Vaporization, Homogenous Flow.

\section{Introduction}

Modelling the two-phase flow through a capillary is of great interest for several decades and has not yet been solved satisfactorily. Capillaries determine the operating point of (rather small) heat pumps where they are widely used as static throttle device. Hence, in order to obtain a predictive overall model of the heat pump, it is crucial to provide a reliable capillary model. Against this background, many researchers focus on the homogenous flow approach with the major challenge to find an appropriate and predictive correlation for the two-phase viscosity. Sempértegui-Tapia and Ribatski [1] criticize that experiments are often inaccurately performed and documented which makes it difficult to identify an appropriate flow model that follows the experimental data. Respectively, there is a lack of precise information concerning the experimental apparatus and/or geometrical parameters of the capillary. Yet, the diameter and the absolute roughness of the capillary have a strong influence on the simulated flow through capillaries as shown in this work.

There is a broad database concerning HFCs though these refrigerants gradually lose their importance due to climate protection. This work contributes to extend the general database on two-phase pressure drop by experimental data of propane as an alternative refrigerant. The gathered experimental data supplements the current database in terms of higher mass fluxes and very precise evaluation of the capillary specification such as diameter and roughness.

A further major focus lies on the hysteresis effect on vaporization delay that becomes obvious in the gathered experimental data. Additionally, the presence of a metastable region affects the measured pressure drop. Many databases lack the documentation of these effects that apply for the pressure drop through a capillary. However, they need to be considered within the capillary model for a predictive behaviour in a dynamic overall system such as a heat pump.

\section{Experimental Apparatus and Precise Capillary Specification}

Fig. 1 shows the schematic diagram of the experimental apparatus that is operated with propane. Via implemented control strategies it is possible to adjust different subcooling temperatures of the refrigerant at the inlet of the capillary (TR1), inlet pressures (PR-1) and mass fluxes of the refrigerant (FIRC-1) in order to gather a comprehensive experimental 
database. The condition of the flow at the capillary inlet is independent of the capillary outlet because the second control valve decouples the inlet from the outlet of the capillary. The design of the experimental apparatus enables a wide range of operating conditions (PR-1: 10-30 bar, TR-1 $20-80^{\circ} \mathrm{C}$, FIRC-1: $2-20 \mathrm{~kg} / \mathrm{h}$ ). The accuracy of the pressure sensors is specified to $\pm 0.7 \%$. Thermocouples of type $\mathrm{T}$ serve for temperature measurement providing an accuracy of $\pm 0.5 \mathrm{~K}$. The Coriolis mass flow meter provides an accuracy of $0.3 \%$.

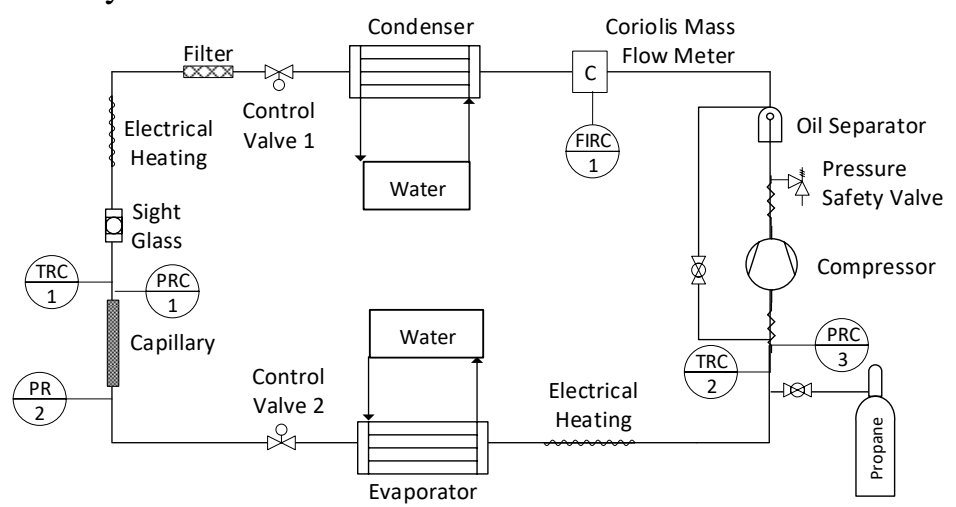

Fig. 1: Schematic Diagram of the Experimental Apparatus.

The manufacturer specifies the considered capillary with an inner diameter ID $=1.1 \mathrm{~mm} \pm 0.02$. There is no statement concerning the absolute roughness $(\mathrm{Ra})$ of the inner surface in general. For modelling the two-phase flow through the capillary, however, this parameter is necessary (see Eq. (2)). A statistical analysis of the ID (investigation with a light microscope of five cut out elements of ten different capillaries of one charge from the same manufacturer) corrected the expected diameter to a value of $\mathrm{ID}=1.095 \mathrm{~mm} \pm 0.009$. Measurements with the white light interferometry indicate that the absolute roughness is $\mathrm{Ra}=0.5-1.5 \mu \mathrm{m}$. Further measurements to provide statistical significance of the absolute roughness are in progress.

\section{Adaptable Homogenous Flow Model}

Eqs. (1) - (3) represent the common homogenous flow model through a capillary tube in order to determine the pressure drop $\Delta p$. The first summand in Eq. (1) indicates the friction pressure drop at the liquid phase with $L_{l i q}$ the length of the liquid phase, $f$ the friction factor, $I D$ the inner diameter of the capillary, $\xi$ the pressure drop coefficient due to sudden contraction at the inlet of the capillary, $\rho$ the density of the refrigerant and $v$ the flow velocity. Analogously, the last summand represents the friction pressure drop at the two-phase region with the length $L_{t p}$ and $G$ the mass flux. The middle summand describes the acceleration pressure drop arising from the change in momentum with $x$ the vapor quality, $\alpha$ the void fraction, $\rho_{v}$ and $\rho_{l}$ the vapor and liquid density, accordingly. Eq. (2) indicates the calculation of the friction factor following the ColebrookWhite equation [3] with $R a$ the absolute roughness of the inner surface of the capillary and $R e$ the Reynolds-number.

$$
\begin{gathered}
\Delta p=\left(L_{l i q} \cdot \frac{f}{2 \cdot I D}+\xi\right) \cdot \rho \cdot v^{2}+\Delta\left\{\frac{G^{2} \cdot x^{2}}{\alpha \rho_{v}}+\frac{G^{2} \cdot\left(1-x^{2}\right)}{(1-\alpha) \cdot \rho_{l}}\right\}+f \cdot \frac{G^{2}}{2 \cdot I D \cdot \rho} \cdot L_{t p} \\
\frac{1}{\sqrt{f}}=1.14-2 \cdot \log \left(\frac{R a}{I D}\right)+\frac{9.3}{R e \cdot \sqrt{f}} \\
R e=\frac{v \cdot I D \cdot \rho}{\mu}
\end{gathered}
$$


A common subject of investigations is to identify an appropriate viscosity correlation $\mu$ for the two-phase flow. Yet, inaccurate capillary specifications can shadow the actual effect of different viscosity correlations, as shown in [2]. Fig. 2 exemplarily illustrates the deviation of the calculated outlet pressure for uncertainties in the inner diameter (ID $=1.1 \mathrm{~mm} \pm$ \pm 0.02 ). Eqs. (1) - (3) together with the viscosity correlation of Beattie and Whalley [4] served as calculation basis.

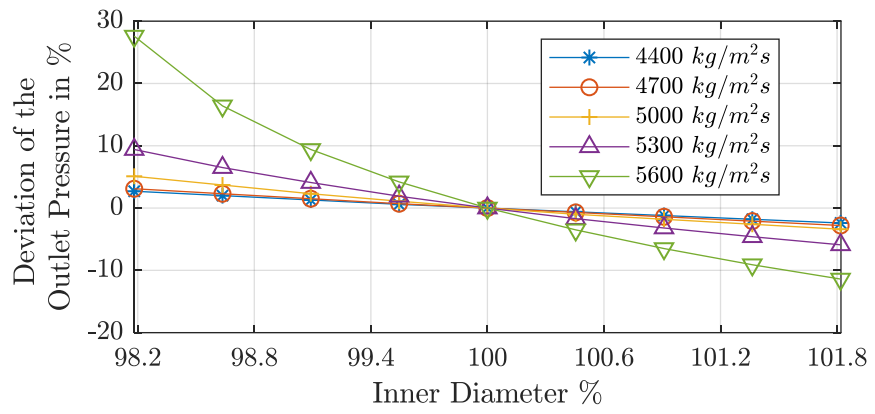

Fig. 2: Deviations of the calculated outlet pressure for deviations of the inner diameter, inlet pressure of 26 bar and subcooling of $15^{\circ} \mathrm{C}$.

The impact of inaccurate diameter specification on the pressure drop increases for higher mass fluxes, as can be seen in Fig. 2, as well as for lower inlet pressures and lower subcooling temperatures which was investigated in addition. In such cases, discrepancies between the numerical and experimental data can erroneously be traced back on inappropriate viscosity correlations. Fig. 2 shows that a deviation of only $1.8 \%$ of the inner diameter can lead to deviations in the outlet pressure of about $30 \%$. Hence, uncertainties of the inner diameter need to be considered in the numerical analysis.

Little research is available that takes into account the hysteresis effect on vaporization as Javidmand outlines in [5]. For this reason, this work additionally contributes to an experimental database of hysteresis curves. On this basis, a memory function needs to be implemented into the equation set above that captures the hysteresis effect.

\section{Experimental Data}

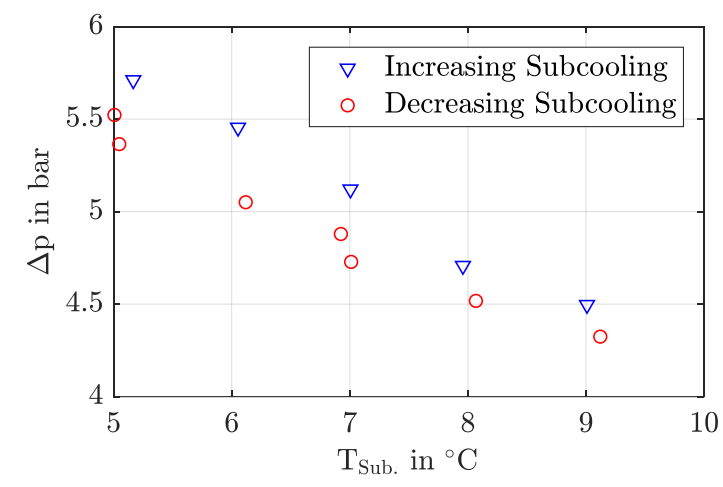

Fig. 3: Hysteresis effect on vaporization delay of propane for inlet pressure of 15 bar and mass flux of $4300 \mathrm{~kg} / \mathrm{m}^{2} \mathrm{~s}$.

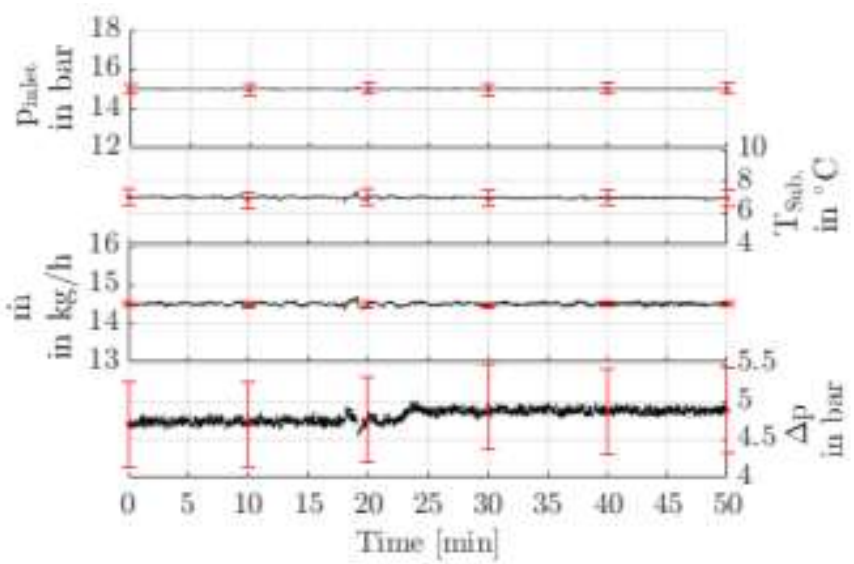

Fig. 4: Collapse of a metastable region.

The presented experimental data in this section exemplarily shows the importance of considering the hysteresis effect and the metastability in order to obtain a valid capillary model. Fig. 3 contrasts exemplary measurements of the pressure drop $\Delta p$ of propane for a constant inlet pressure $p_{\text {inlet }}$ of $15 \mathrm{bar}$, constant mass flux of $4300 \mathrm{~kg} / \mathrm{m}^{2} \mathrm{~s}(\hat{=} 14.5 \mathrm{~kg} / \mathrm{h})$ and various subcooling temperatures $T_{\text {Sub. }}$. Stationary operation of each recorded measurement point is at least 20 minutes. It shows that the order of measurements (increasing or decreasing subcooling) has an effect on the magnitude of the recorded pressure drop. Without a hysteresis, the pressure drop should be similar for similar operation conditions. This is not the case and so the hysteresis effect is obvious. Starting from a low subcooling temperature and subsequently increasing the subcooling 
temperature results in a higher pressure drop (by an average of $5 \%$ ) than vice versa. It is further remarkable that for the decreasing subcooling measurements the pressure drop at $5{ }^{\circ} \mathrm{C}$ and $7{ }^{\circ} \mathrm{C}$ subcooling was not stable. Fig. 3 indicates a discontinuous change of pressure drop at these operating points. Fig. 4 shows the time resolved experimental data of the pressure drop for $7{ }^{\circ} \mathrm{C}$ subcooling in the decreasing subcooling curve. It can be seen that, overall, the inlet conditions remain the same but there is a change in the recorded pressure drop after approximately 23 minutes. Meyer and Dunn [6] interpreted this kind of behaviour as the collapse of a metastable region. It is possible that the short period of irregularity in mass flux $\dot{m}$ between 18-20 minutes led to the collapse, which thereupon caused an increase in pressure drop.

A predictive flow model has to capture the measured effects. This requires comprehensive experimental data on hysteresis curves and metastability effects at different operating conditions that are to be shown by measurements for a wide operating area with the presented experimental apparatus.

\section{Conclusion}

Much attention is paid to predict the pressure drop through a capillary, since capillaries are a commonly used throttle device in heat pumps. Many researchers focus on the homogenous flow approach and strive to find an appropriate and predictive correlation for the two-phase viscosity. However, inaccurate experimental setups and imprecise capillary specifications that are part of the flow model have a superior influence on the simulated pressure drop than a variation or adaption of common viscosity correlations. Consequently, a thorough experimental setup and a precise determination of the capillary specification is inevitable for accurate experimental and numerical comparison of the flow through a capillary. In this work, significant measurements have been carried out to determine the needed parameters more precisely than delivered by the manufacturer of the capillary.

Little data exists for the two-phase pressure drop of natural refrigerants compared to data for HFCs as mentioned in [1]. What is more, even less data exists that takes into account the hysteresis effect on vaporization delay. Experimental investigations of the pressure drop of propane for various subcooled inlet conditions show that the hysteresis has a significant impact on the pressure drop (see Fig. 2). Hence, when modelling the flow through a capillary, this effect needs to be taken into account. Meyer and Dunn [6] recommended incorporating the hysteresis effect for capillary models that are part of an overall heat pump model. Depending on the operating condition of the heat pump, the inlet condition at the capillary changes. Therefore, the hysteresis curve of the capillary is passed through and must have an influence. To the best of the author's knowledge this recommendation has not or not a lot been followed in the past. This work contributes to the database of the hysteresis and metastability effects in a capillary tube so that they can be considered in the numerical model of the flow. Therefore, comprehensive experimental investigations on hysteresis curves for different operating conditions are to be performed. Since the experimental apparatus is flexible, numerous inlet pressures, subcooling temperatures and mass fluxes will be investigated. Additionally, the experimental setup allows experiments with the alternative fluid isobutene to extend the database of natural refrigerants for an extensive investigation of the considered effects for similar refrigerants.

\section{References}

[1] D. F. Sempértegui-Tapia and G. Ribatski, "Two-phase frictional pressure drop in horizontal micro-scale channels: Experimental data analysis and prediction method development," Int. J. Refrigeration, vol. 79, pp. 143-163, 2017.

[2] X. Gabrisch et al., "Dynamische Modellierung einer Kältemittel-Luft-Wärmepumpe," in Proceedings of the Deutsche Kälte- und Klimatagung, Bremen, 2017.

[3] C. F. Colebrook, "Turbulent flow in pipes, with particular reference to the transition region between the smooth and rough pipe laws," J. Inst. Civil Eng., vol. 11, no. 4, pp. 133-156, 1939.

[4] D. R. H. Beattie and P. B. Whalley, "A Simple Two-Phase Frictional Pressure Drop Calculated Method," Int. J. Multiphase Flow, vol. 8, no. 1, pp. 83-87.

[5] P. Javidmand, "Quasi-one-dimensional numerical analysis of choked two-phase flashing flow through millitubes," Ph.D. Dissertation, Dept. Aerospace Eng., Wichita State Univ., Wichita, KS.

[6] J. J. Meyer and W. E. Dunn, "New Insights into the Behavior of the Metastable Region of an Operating Capillary Tube," HVAC\&R Research, vol. 4, no. 1, pp. 105-115, 1998. 